\title{
VARIABILITÉ À MOYEN TERME DES PEUPLEMENTS DE POISSONS DES COURS D'EAU BAS-NORMANDS.
}

\section{BELKESSAM et T. OBERDORFF (auteur pour correspondance)}

Muséum National d'Histoire Naturelle, Laboratoire d'Ichtyologie Générale et Appliquée, 43 rue Cuvier, 75231 Paris Cedex 5, France.

\section{RÉSUMÉ}

Dans l'optique de comprendre quels sont les processus qui gouvernent la structure des peuplements de poissons des cours d'eau bas-normands, une première approche descriptive est réalisée à travers l'analyse de la variabilité temporelle de ces peuplements. Les données exploitées portent sur 14 stations situées sur 10 cours d'eau de Basse-Normandie, échantillonnés de 1990 à 1996.

Les principaux résultats de l'étude sont les suivants :

1. on note, pour l'ensemble du peuplement, un maintien de la composition spécifique au cours du temps (persistance) ;

2. le peuplement présente une structure relativement stable ;

3. les densités moyennes des populations qui composent ce peuplement présentent, en revanche, une importante variabilité temporelle. Cette variabilité est déterminée, en partie, par les variations des débits, lesquelles sont d'autant plus importantes que l'on se situe en aval du cours d'eau ;

4. contrairement à l'hypothèse de HORWITZ (1978) (« extermination hypothesis "), le turnover des espèces n'augmente pas avec un accroissement de la variabilité du peuplement ;

5. la variabilité du peuplement augmente avec le temps.

La position des peuplements de poissons des cours d'eau bas-normands sur le gradient “ stochastique-déterminé » est discutée.

\section{MEDIUM-TERM VARIABILITY OF FISH ASSEMBLAGES IN STREAMS OF THE NORTH-WESTERN FRANCE.}

\begin{abstract}
In order to outline processes governing fish assemblages in streams of the North-Western France, a first descriptive study is realized through the analysis of their temporal variability. Studied data concern 14 sites within 10 different streams sampled annually over the seven-year period from 1990 to 1996 .
\end{abstract}


Salient results are :

1. the assemblage shows a constant species composition (persistence);

2. the assemblage has a relatively stable structure ;

3. the mean densities of the populations are highly fluctuating. These fluctuations are explained, in part, by flow variations, which are more important in downstream areas ;

4. the "extermination hypothesis" (HORWITZ, 1978) is not supported by our data set ;

5. assemblage variability increases with time.

Finally, the position of these fish assemblages along a "stochastic-deterministic" gradient is discussed.

\section{INTRODUCTION}

La gestion des ressources aquatiques, qu'il s'agisse de gestion des stocks ou de l'évaluation de la qualité biologique des milieux, peut se faire à l'aide d'outils tels que les modèles prédictifs ou les indices biotiques. L'utilisation des ces outils requiert la maîtrise des processus essentiels qui gouvernent la structure et/ou le fonctionnement des ensembles considérés. Ceux-ci ne peuvent donc être appliqués qu'à des systèmes dont la variabilité spatio-temporelle reste contrôlable, c'est-à-dire présentant une structure " déterminée ". On peut considérer qu'un peuplement de poissons (défini ici comme l'ensemble des espèces présentes sur une station de pêche) est " déterminé " s'il présente une structure constante en l'absence de modifications importantes des paramètres environnementaux ou encore si sa variabilité suit celle d'un ou de plusieurs de ces paramètres. Au contraire, le peuplement est dit "stochastique " s'il varie de manière imprévisible même si les conditions environnementales sont constantes ou varient autour d'une moyenne. Dans le cas des peuplements se trouvant dans des milieux présentant une forte variabilité interannuelle (cas des peuplements de poissons), il est difficile d'évaluer leur degré de variabilité intrinsèque, ce qui explique, en partie, le grand nombre d'études réalisées sur ce sujet et les diverses polémiques qui ont suivi entre les adeptes de l'" école déterministe " et ceux de l'« école stochastique ». L'« école déterministe » soutient que les peuplements sont en général stables (ou en état d'équilibre ou de succession) parce que l'abondance des espèces est régulée par des processus déterminés tels que la prédation, la compétition ou le partage des ressources et ce, malgré la variabilité des facteurs environnementaux du cours d'eau (GORMAN et KARR, 1978 ; GATZ, 1979 ; MOYLE et VONDRACEK, 1985). L'“ école stochastique " soutient que les facteurs environnementaux sont trop souvent instables pour permettre l'équilibre, de sorte que l'abondance des espèces est largement déterminée par des changements environnementaux imprévisibles plutôt que par des interactions biologiques. Ainsi, GROSSMAN et al. (1982) ont tenté de décrire les mécanismes qui régulent l'abondance et la structure des poissons d'un fleuve de l'Indiana (USA) et ont conclu à un manque de stabilité du peuplement et ce, en l'absence de modifications importantes du milieu. IIs attribuent cette variabilité temporelle, apparemment aléatoire, de la communauté de poissons aux fluctuations saisonnières et interannuelles des facteurs hydrologiques. Leur étude a été contestée (HERBOLD, 1984 ; YANT et al., 1984 ; ROSS et al., 1985 ; MEFFE et BERRA, 1988) ce qui a, entre autres, permis de démontrer que le peuplement était régulé par des processus déterminés mais répondait à des paramètres environnementaux stochastiques (YANT et al., 1984). Lors d'une étude sur les peuplements de poissons d'Afrique de l'Ouest, HUGUENY (1992) constate que des variations du milieu identiques conduisent à des changements similaires dans les peuplements. Par ailleurs, 
SCHLOSSER $(1982,1987,1990)$ suggère que les rôles relatifs des facteurs biotiques ou abiotiques influant sur la stabilité du peuplement sont susceptibles de varier selon le gradient longitudinal des cours d'eau.

Dans la présente étude, nous décrirons la variabilité temporelle des peuplements de poissons des cours d'eau bas-normands en essayant de tester les hypothèses suivantes :

1. les peuplements sont-ils persistants, c'est-à-dire observe-t-on un maintien des espèces au cours du temps ? Si oui, cette persistance est-elle accompagnée d'une stabilité du peuplement telle que l'ont définie GROSSMAN et al. (1990) : constance des densités au cours d'une période supérieure au turnover moyen des espèces ? Si tel est le cas, le peuplement peut être considéré comme déterminé ;

2. la variabilité du peuplement n'est-elle pas associée à celle des conditions environnementales ? Si tel est le cas, le peuplement est donc gouverné par des processus environnementaux variables mais déterminés et présente donc une structure déterminée ;

3. plus le peuplement présente une forte variabilité, plus les risques d'extinction des espèces sont importants et plus le turnover des espèces augmente : "extermination hypothesis " (HORWITZ, 1978) ;

4. à l'échelle de la population ou du peuplement, la variabilité augmente avec le temps (PIMM et REDFEARN, 1988 ; BENGTSSON et al., 1997). En d'autres termes, existe-t-il une relation positive entre les indices de variabilité annuelle des peuplements et la durée de suivi?

\section{MATÉRIEL ET MÉTHODES}

\section{Origine et collecte des données}

Nous avons utilisé les résultats de pêche de 14 stations non ou peu perturbées, situées sur 10 cours d'eau différents, issus des campagnes de prospection du Réseau Hydrobiologique et Piscicole (R.H.P.) de Basse-Normandie, gérées par le Conseil Supérieur de la Pêche (Figure 1). La sélection de ces stations témoins a été réalisée sur la base : (1) de la qualité physico-chimique de l'eau relevée sur les cartes de qualité des Agences de l'Eau durant l'année d'échantillonnage (seules les stations de qualité physico-chimique bonne ou moyenne - classes $1 \mathrm{~A}, 1 \mathrm{~B}$ et 2 - ont été retenues) et (2) de la connaissance de l'habitat physique de chacune des stations. Les facteurs considérés afin de juger de l'habitat physique incluaient la morphologie du chenal, son degré d'anthropisation, le régime des débits, la qualité des berges et du substrat, les conditions d'écoulement et la représentativité du site dans la région. Les stations témoins sélectionnées ne sont bien évidemment quasiment jamais exemptes d'impacts liés aux activités humaines mais restent les moins anthropisées de la région considérée. Ces stations ont été prospectées de 1990 à 1996. Pour l'ensemble des stations, l'échantillonnage a été réalisé par pêche électrique. Deux passages successifs sont effectués avec retrait des poissons capturés au premier passage. La longueur de chaque station, entre 150 et $200 \mathrm{~m}$, couvre le preferendum d'habitat de l'ensemble des espèces (KENNEDY et PITCHER, 1975 ; NICOLAS et al., 1994 ; MINNS, 1995). Une fois les individus identifiés, mesurés et pesés, ils sont relâchés. L'estimation du peuplement est ensuite réalisée en appliquant la méthode de CARLE et STRUB (1978). A l'issue de ces opérations, les informations recueillies sont les suivantes : richesse spécifique de la station (Richesse Spécifique Locale ou RSL), densité estimée de chacune des espèces (nombre d'individus de l'espèce pour $100 \mathrm{~m}^{2}$ prospectés). 


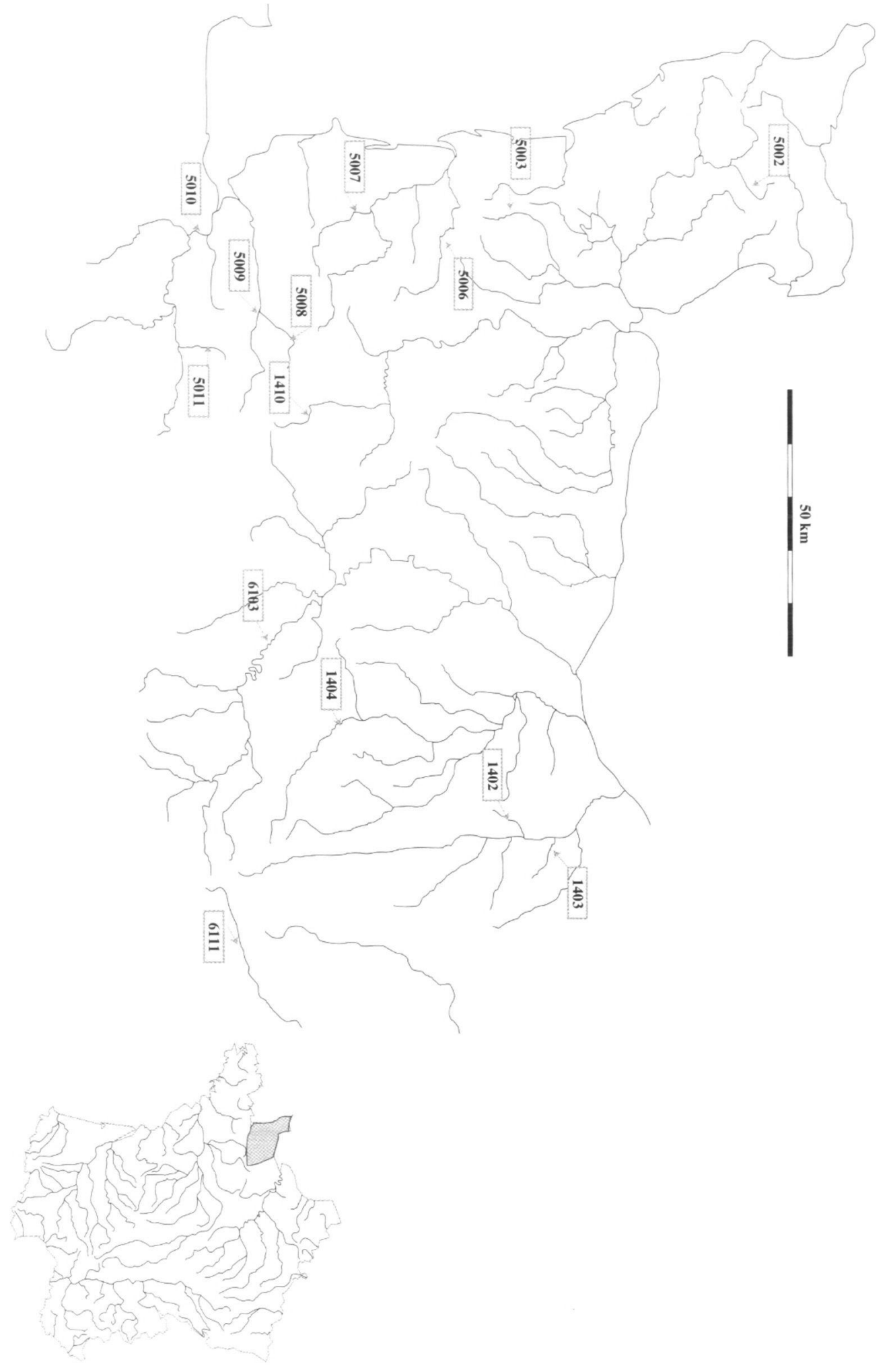

Figure 1

Position géographique des stations de pêche analysées.

Figure 1

Position of the studied sites. 
En parallèle, sont relevées les caractéristiques relatives à la position de la station sur le gradient longitudinal du cours d'eau (i.e. superficie du bassin versant drainé, distance aux sources, pente, altitude). A partir des données de températures (source : Météo France région Basse-Normandie) et des données de débits (source : DIREN Basse-Normandie), des valeurs moyennes annuelles ont été calculées (Tmoy et Dmoy). Certaines stations n'étant pas renseignées sur les débits, nous avons retenu les données de débits disponibles au plus près de la station. Les données de précipitations (source: Météo France) correspondent aux moyennes annuelles. La liste des paramètres des stations renseignées figure dans le Tableau I.

\section{Tableau I}

\section{Localisation et description des 24 stations étudiées.}

\section{Table I}

\section{Position and description of the 24 studied sites.}

\begin{tabular}{|c|c|c|c|c|c|c|c|c|c|c|}
\hline STATION & BASSIN & COUAS D'EAU & CONMUNE & $\begin{array}{c}\text { OSTANCE } \\
\text { AUX } \\
\text { SOURCES } \\
(\mathrm{km})\end{array}$ & ALTITUDE & $\begin{array}{c}\text { SUPERFICIE } \\
\text { DU BASSIN } \\
\text { VERSANT } \\
\text { DRANE } \\
\text { (kTH } 2)\end{array}$ & $i_{k+1}$ & $\begin{array}{c}\text { CV } \\
\text { OEBI } \\
\text { ANWUEL } \\
\text { MOYEN }\end{array}$ & $\begin{array}{l}\text { CV } \\
\text { TEMPE AATURES } \\
\text { ANNUELLES } \\
\text { MOYENNES }\end{array}$ & $\begin{array}{c}\text { CV } \\
\text { PAECIPTA TIONS } \\
\text { ANNUELLES } \\
\text { MOYENNES }\end{array}$ \\
\hline 1402 & TOVOUES & PAE DAUGE & MANERBE & 5 & 60 & 9.5 & 9.2 & 18.09 & 762 & 16.13 \\
\hline 1403 & TOUOUES & CHAUSSEY & BLANGY.LE.CHATEAU & 6.5 & 40 & 30 & 6.1 & 18.09 & 7,62 & 16,13 \\
\hline 1404 & DIVES & DIVES & BEAUMAIS & 31.5 & 55 & 290.5 & 2,2 & 61,83 & - & - \\
\hline 1410 & VIRE & VIAE & TRUTTEMER-LE-GRAND & 4 & 240 & 7 & 6.7 & 27.55 & 5.49 & 17.21 \\
\hline 5002 & DOUVE & GLOIRE & TAMERVILLE & 9 & 50 & 29 & 5 & . & 4.83 & 20.08 \\
\hline 5003 & TAuTE & TAUTE & ST-SAUVEUR-LENDELIN & 7.5 & 35 & 25 & 5 & 32.47 & 5.7 & 18.85 \\
\hline 5006 & SIENNE & SIENNE & HAMBYE & 34.5 & 55 & 201 & 3 & 41.76 & 5.7 & 18.85 \\
\hline 5007 & SIENNE & AIROU & LE MESNIL.ROGUES & 17 & 45 & 89 & 5 & 38.73 & 4.88 & 19.34 \\
\hline 5008 & SEE & SEE ROUSSE & SOURDEVAL & 5.5 & 190 & 26 & 10 & 18.13 & 5.7 & 18.85 \\
\hline 5009 & SEE & SEE & CHERENCE-LE-ROUSSEL & 16 & 80 & 86 & 12.5 & 18.13 & 7.23 & 24.62 \\
\hline 5010 & SELUNE & SELUNE & DUCEY & 56 & 15 & 759 & 4 & 63.75 & 7.23 & 24.62 \\
\hline 5011 & SELUNE & CANCE & FOMAGNY & 11 & 120 & 36.8 & 20 & 18.13 & 7.23 & 24.62 \\
\hline 6103 & OANE & FONTAINE AU HERON & STE-CROIX-SUR-ORNE & 4 & 150 & 11 & 10 & 35.26 & 6.97 & 21,64 \\
\hline 6911 & RISLE & RISLE & AUBE & 16 & 220 & 115 & 0.7 & 31.67 & . & - \\
\hline
\end{tabular}

\section{Méthodes d'analyse}

La persistance a été mesurée à partir de l'index de turnover (renouvellement) faunistique (DIAMOND et MAY, 1977 ; MEFFE et BERRA, 1988). Le turnover entre deux échantillons est mesuré à partir du rapport $T=(C+E) /(S 1+S 2), C$ et $E$ étant respectivement le nombre de colonisation et d'extinction des espèces durant la période d'étude, $S 1$ et $\mathrm{S} 2$ le nombre d'espèces de chaque échantillon. T varie de 0 , pas de turnover, à 1, turnover total. Un $T$ moyen a été calculé sur les 7 années pour chaque station. La persistance du peuplement de chaque station est donnée par $P=1-T$ et varie de 0 , pas de persistance, à 1 , persistance complète.

Le niveau typologique de chaque station a été déterminé à partir de la typologie proposée par VERNEAUX $(1977,1981)$ et seules les espèces caractéristiques de ces milieux (capables de maintenir des populations viables dans les sites étudiés) ont été retenues pour l'étude de la stabilité. Par ailleurs, afin d'éliminer l'effet des espèces rares dans la variabilité du peuplement, les espèces qui n'étaient pas présentes au moins 4 années sur 7 ont été écartées de ce jeu de données (HUGUENY et al., 1995).

La stabilité (maintien des densités des espèces au cours du temps) des peuplements a été analysée en calculant divers types de coefficients de variation (CV) ainsi que le W (coefficient de corrélation de rang) de KENDALL (SIEGEL, 1956). Ces deux descripteurs sont, à notre connaissance, les seuls actuellement disponibles pour quantifier la variabilité temporelle des peuplements (GROSSMAN et al., 1990). Le CV est donné par 
la formule : $\mathrm{CV}=$ (écart type $/$ moyenne $) \times 100$. Nous avons ensuite apprécié le degré de stabilité des peuplements, en se basant sur la classification proposée par FREEMAN et al. (1988) : (1) CV $\leq 25 \%=$ fortement stable ; (2) $25 \%<\mathrm{CV} \leq 50 \%$ = modérément stable ; (3) $50 \%<\mathrm{CV} \leq 75 \%=$ modérément fluctuant ; (4) CV $>76 \%=$ fortement fluctuant. Cette classification a été appliquée aux CV de la densité totale des peuplements (CVDT) et aux $\mathrm{CV}$ de la densité moyenne des peuplements (CVDM).

W est une méthode statistique non paramétrique d'analyse des corrélations basée sur les rangs de densités (d'abondances) des espèces. Elle permet de classer les espèces par rang d'abondance (de densité), $W$ varie de 0 (pas de relation entre les densités des différentes années) à 1 (concordance totale dans les rangs de densités entre les années), (BENGTSSON et al., 1997).

Les divers indices de stabilité calculés sont donc

CVRSL : CV calculé à partir des richesses spécifiques pour chaque station ;

CVDT : CV calculé à partir de la densité totale du peuplement ;

CVDM : pour chaque station, des CV moyens ont été calculés à partir des CV des densités de chaque espèce (CVSP) ;

W : coefficient de rang de KENDALL calculé par station à partir des densités des espèces ;

CVDMP : afin de tester l'effet de la durée d'étude sur la variabilité temporelle des peuplements, nous avons comparé les valeurs des CVDM obtenues sur 7 années d'études (données complètes) à celles obtenues seulement durant 4 années (données partielles: CVDMP). Si la durée de l'étude a un effet positif sur la variabilité temporelle des peuplements, on doit obtenir des CVDM supérieurs aux CVDMP (Hypothèse 1). Sur 24 stations, on en recense 17 totalement renseignées (7 années). En comptant le nombre de stations pour lesquelles les CVDM des données complètes sont supérieurs aux CVDM des données partielles, sous $\mathrm{HO}$ ce nombre doit être inférieur à $50 \%$ des stations (soit 8,5) ; inversement, il doit être significativement supérieur à $50 \%$ sous $\mathrm{H} 1$;

WP : dans le cas des estimations à l'aide du W, sous $\mathrm{HO}, \mathrm{W}$ des données complètes est supérieur à WP (données partielles) et inversement sous H1.

Les relations entre la variabilité temporelle des peuplements et les paramètres caractéristiques du gradient longitudinal (altitude, superficie du bassin versant drainé, pente), les facteurs climatiques (température, débit, précipitation) et la persistance, ont été analysés à partir de modèles de régressions simples et multiples.

\section{RÉSULTATS}

Les valeurs des divers indices de variabilité figurent dans le Tableau II.

Pour l'ensemble des stations, l'étude de la persistance des espèces révèle un maintien des richesses spécifiques au cours des 7 années considérées ( $P$ moyen $=0,90$ ). Le CVRSL moyen $(13,68)$ confirme cette caractéristique des peuplements. Selon le schéma empirique de FREEMAN et al. (1988), la densité totale du peuplement est modérément stable (CVDT moyen $=48,49$ ) et leur densité moyenne modérément à fortement fluctuante (CVDM moyen $=75,33$ ). En revanche, la variabilité temporelle du peuplement calculée sur les densités des espèces au moyen du $W$ de KENDALL met en évidence une plus grande stabilité ( $W$ moyen $=0,62$ ). 


\section{Tableau II}

Indices de variabilité retenus.

$P$ : persistance ; CVRSL : coefficient de variation de la richesse spécifique locale ; CVDT : coefficient de variation des densités totales des espèces ; CVDM : coefficient de variation moyen des densités ; $W$ : coefficient de rang de KENDALL ; CVDMP : coefficient de variation moyen des densités, données partielles ; WP : coefficient de rang de KENDALL des données partielles.

Table II

Indices of variability retained.

$P$ : persistence ; CVRSL : CV of local species richness ; CVDT : CV of total species densities ; CVDM : mean CV of populations densities. W : KENDALL's coefficient of concordance ; CVDMP : mean CV of populations densities, partial data. $W$ : KENDALL's coefficient of concordance, partial data.

\begin{tabular}{ccccccccc}
\hline STATION & P & CVRSL & CVDT & CVDM & W & CVDMP & WP & RSM \\
\hline 1402 & 0,95 & 9,80 & 45,78 & 37,02 & 1,00 & 27,09 & 1,00 & 3,90 \\
1403 & 0,93 & 14,43 & 43,37 & 62,29 & 1,00 & 49,98 & 1,00 & 4,00 \\
1404 & 0,93 & 10,37 & 91,61 & 95,26 & 0,60 & 88,86 & 0,72 & 7,30 \\
1410 & 0,91 & 14,21 & 36,91 & 80,44 & 0,75 & 68,52 & 0,69 & 4,90 \\
5002 & 0,82 & 12,90 & 42,34 & 63,53 & 0,33 & 55,89 & 0,89 & 5,80 \\
5003 & 1,00 & 0,00 & 48,51 & 71,81 & 0,54 & 49,47 & 0,78 & 6,00 \\
5006 & 0,98 & 4,15 & 71,73 & 88,48 & 0,54 & 90,62 & 0,56 & 9,80 \\
5007 & 0,89 & 14,38 & 64,03 & 105,37 & 0,84 & 92,31 & 0,90 & 8,70 \\
5008 & 0,97 & 6,45 & 41,24 & 72,40 & 0,50 & 51,73 & 0,69 & 5,90 \\
5009 & 0,87 & 19,61 & 28,69 & 52,24 & 0,33 & 50,10 & 0,57 & 8,20 \\
5010 & 0,71 & 37,67 & 71,85 & 140,43 & 0,48 & 125,87 & 0,55 & 9,30 \\
5011 & 0,88 & 17,25 & 31,05 & 68,81 & 0,18 & 49,48 & 0,44 & 6,60 \\
6103 & 0,87 & 21,96 & 31,32 & 56,29 & 0,86 & 64,60 & 0,82 & 3,10 \\
6111 & 0,83 & 8,31 & 30,43 & 60,24 & 0,72 & 62,71 & 0,70 & 6,40 \\
\hline Moyenne & 0,90 & 13,68 & 48,49 & 75,33 & 0,62 & 66,23 & 0,74 & 6,42 \\
\hline Ecart type & 0,08 & 9,23 & 19,80 & 26,91 & 0,26 & 25,94 & 0,18 & 2,14 \\
\hline
\end{tabular}

On note une relation significative entre la variabilité temporelle des densités moyennes du peuplement (CVDM) et, d'une part, trois des paramètres du gradient longitudinal (superficie du bassin versant drainé, distance aux sources, pente (Tableau III)) et, d'autre part, les variations de débits (Tableau III et Figure 2). 


\section{Tableau III}

Résultats des régressions simples des indices de variabilité (CV, W) en fonction des variables environnementales.

NS : non significatif $;^{\star}: p<0,05 ;{ }^{\star \star}: p<0,01 ;{ }^{\star \star \star}: p<0,001$.

Table III

Results of simple regressions analyses of variability indices (CV, W) versus environmental variables.

NS : non significant $;^{\star}: p<0.05 ;^{\star \star}: p<0.01 ;{ }^{\star \star \star}: p<0.001$.

\begin{tabular}{lcl} 
& CVDM & W \\
\hline Superficie du bassin versant drainé $(\log )$ & $(+)^{\star \star}$ & NS \\
Distance aux sources $(\log )$ & $(+)^{\star \star}$ & NS \\
Pente (log) & NS & NS \\
CV débit moyen annuel & $(+)^{\star \star \star}$ & NS \\
\hline
\end{tabular}

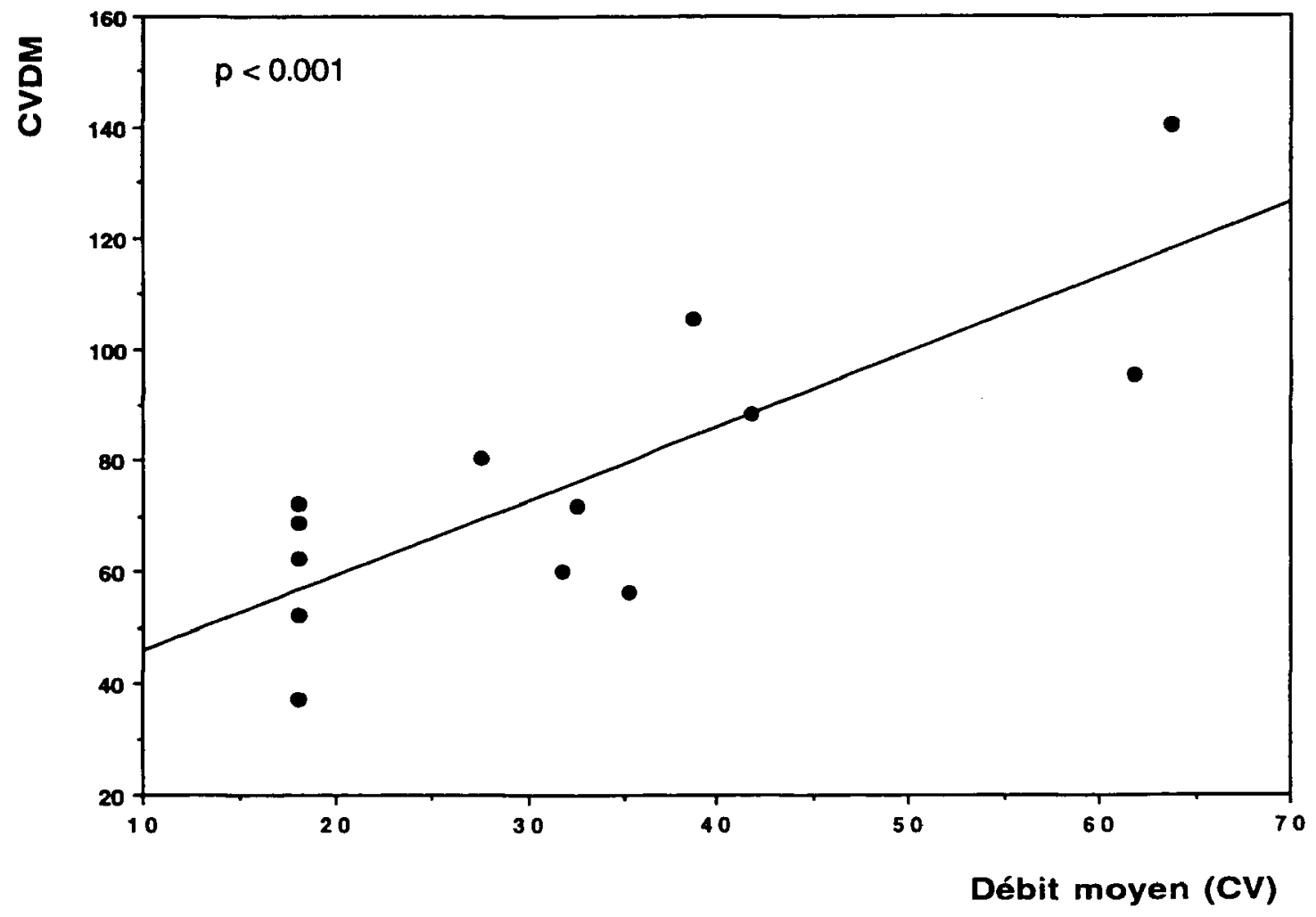

\section{Figure 2}

Relation entre les CVDM et les CV des débits moyens.

\section{Figure 2}

Relationship between CVDM and mean flows CV. 
Pour tester la relation entre la variabilité du peuplement et la persistance des espèces (hypothèse de HORWITZ, 1978), nous avons éliminé l'effet du gradient longitudinal sur les CVDM en réalisant une régression multiple entre les CVDM et les paramètres du gradient longitudinal et retenu les résidus des CVDM. Le modèle de régression linéaire entre les résidus des CVDM et la persistance ne met en évidence aucune relation significative (Figure 3 ).

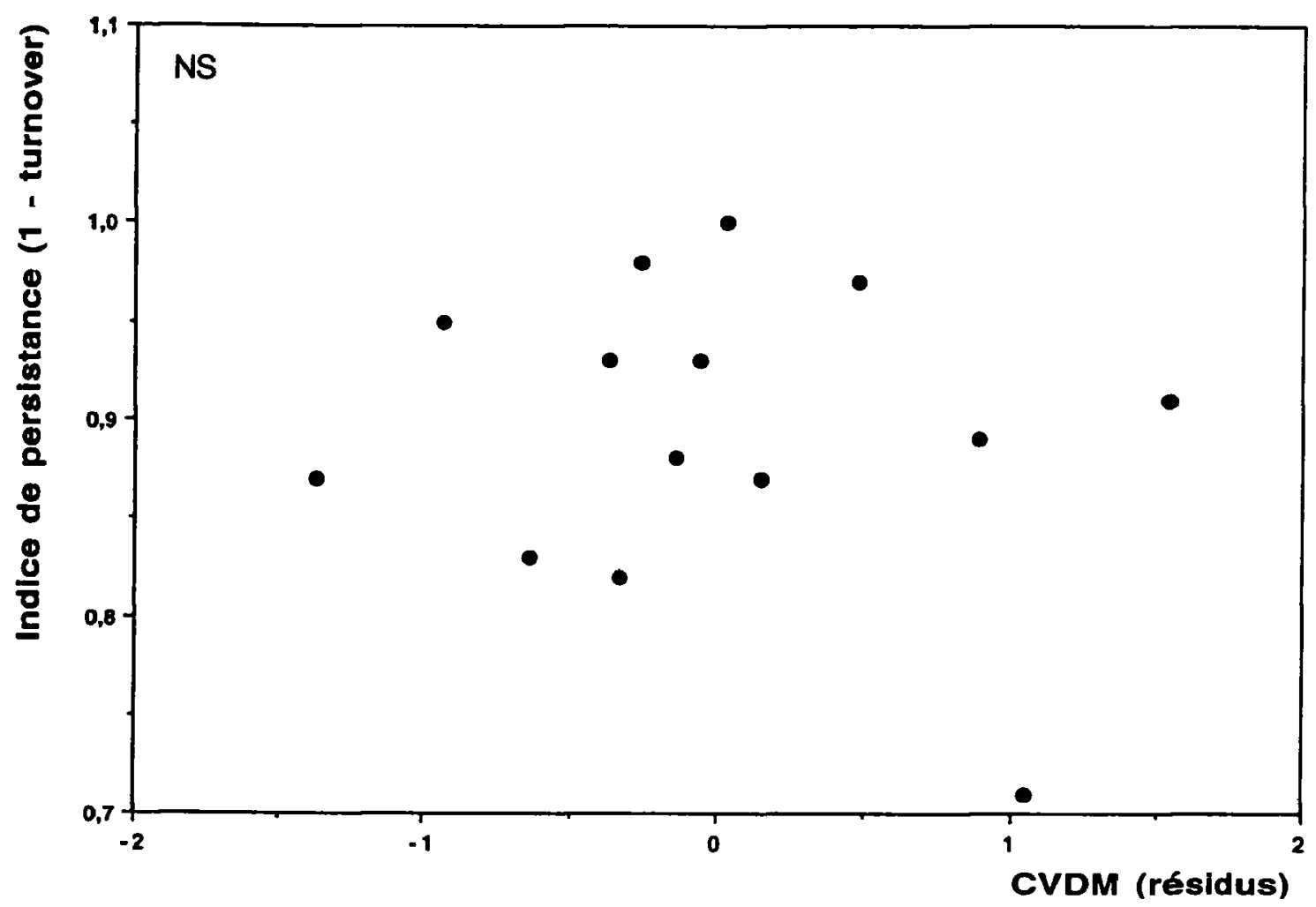

Figure 3

Relation entre les résidus des CVDM et l'indice de persistance (1-T).

Figure 3

Relationship between CVDM (residual) and the persistence indice (1-T).

$85 \%$ des stations ont un CVDMP inférieur au CVDM, et $69 \%$ des stations ont un WP supérieur au W. L'hypothèse $\mathrm{H} 1$ est donc acceptée : la variabilité temporelle augmente lorsque l'on augmente le nombre d'années d'études.

\section{DISCUSSION}

Comme en témoignent les valeurs de persistance ( $P$ moyen $=0,90)$ et de manière indirecte les CVRSL (moyenne $=13,68$ ), on note un maintien des espèces au cours du temps (Tableau I). Si l'on applique la classification de FREEMAN et al. (1988) aux CVDT et aux CVDM, la densité totale des peuplements est modérément stable (CVDT moyen $=48,49$ ) alors que leur densité moyenne est modérément à fortement fluctuante (CVDM moyen $=75,33$ ). Il est probable qu'une partie de cette variabilité soit liée 
à la technique d'échantillonnage utilisée qui n'estime qu'imparfaitement les densités des petites espèces. Cela étant, les quelques études conduites sur les peuplements de poissons de cours d'eau aboutissent à des résultats similaires. Ainsi, HUGUENY et al. (1995), dans une étude de la variabilité temporelle de 18 peuplements de poissons de rivières françaises et ouest-africaines, obtiennent des valeurs de coefficients de variations relativement proches de celles obtenues dans la présente étude (CVDT $=71$, CVDM = 107,2). De même GROSSMAN et al. (1990), lors d'une étude portant sur 10 rivières du Midwest des USA, obtiennent des valeurs de CVDM comprises entre 70 et 135 (moyenne $=96$ ). En revanche, l'analyse de la variabilité temporelle des peuplements à l'aide du $W$ de KENDALL suggère une plus grande stabilité de ces peuplements $(W$ moyen $=0,62)$, comparée aux résultats obtenus à l'aide des CV. De telles différences ont aussi été observées par GROSSMAN et al. (1990). Si l'on compare les méthodes de calcul des $C V$ à celles des $W$, on observe que ces deux indices ne mesurent pas la stabilité du peuplement de la même façon. En effet, $W$ classe les espèces par rang d'abondance au cours des années, il ne tient compte que de la position de l'espèce dans le peuplement et non des variations réelles de densité. II mesure donc la variabilité au niveau de la structure du peuplement. Néanmoins, il possède certaines limites méthodologiques qui affectent l'interprétation des résultats obtenus (GROSSMAN et al., 1985 ; RAHEL et al., 1984 ; GROSSMAN et al., 1990). En effet, il ne permet de ranger le peuplement qu'en deux groupes : sous $\mathrm{HO}, \mathrm{W}$ n'est pas significativement différent de 0 ; sous $\mathrm{H} 1$, il est significativement différent de 0 . Ainsi, si l'on rejette $\mathrm{HO}$, on admet que le peuplement est stable. II conviendrait de déterminer, comme pour le $\mathrm{CV}$, une classification plus fine des degrés de stabilité, par exemple $0<W<0,25$ : peuplement fortement fluctuant ; $0,25<W<0,5$ : peuplement moyennement fluctuant $; 0,5<W<0,75$ : peuplement moyennement stable $; 0,75<W<1$ : peuplement fortement stable.

Le CV possède également certaines limites qu'il convient de mentionner. En calculant les CVDM, on décompose les peuplements en populations. Ainsi, on n'examine pas réellement le comportement du peuplement mais plutôt la variabilité moyenne des différentes populations qui le composent. Par ailleurs, le CV ne permet pas de déceler la dépendance de la variation de la population avec le temps (augmentations ou diminutions à long terme ou fluctuations cycliques ou tendances corrélées entre peuplements (GROSSMAN et al., 1990)). Le calcul d'un coefficient de variation moyen (CVDM) à partir d'autres coefficients de variation n'a pas réellement de sens sur le plan statistique. En effet, CVMD étant le résultat de rapports de moyennes, et compte tenu de la corrélation entre variance et moyenne, il ne possède pas les propriétés des distributions statistiques usuelles. Le CVDM ne peut donc être utilisé que comme un indicateur empirique de variabilité des différentes populations composant le peuplement.

Si l'on compare la variabilité temporelle des poissons à celle des autres grands groupes d'animaux, on note que, quel que soit le descripteur utilisé, ces derniers sont beaucoup plus stables : CV compris entre 10 et 70 pour les populations d'oiseaux des zones tempérées, et environ 50 pour celles des insectes et des petits mammifères, (PIMM, 1991). W varie de 0,8 à 0,89 pour les communautés animales des sols forestiers (BENGTSSON, 1994) et $W=0,75$ pour les oiseaux terrestres de Grande-Bretagne (BENGTSSON et al., 1997). Ces derniers sont pourtant connus comme ayant des populations fortement fluctuantes. II semblerait que, du fait de leur importante vagilité, ceux-ci soient moins affectés par les variations des conditions environnementales qui, quoi qu'il en soit, restent moins importantes en milieu terrestre qu'en milieu aquatique.

Les régressions simples établies entre les indices de variabilité et les paramètres hydroclimatiques mettent en évidence une relation positive entre les CVDM et les CV des débits annuels moyens $\left(R^{2}=0,64, p=0,001\right.$, Figure 2). La structure du peuplement est donc déterminée, en partie, par les variations des conditions hydrologiques. Les grandes 
variations de débits ont une influence prépondérante sur le succès de recrutement des espèces. Les dommages causés aux frayères, aux oeufs et aux juvéniles de poissons, suite à des périodes de crues et/ou d'étiages, diminuent les densités des années suivantes. On assiste ainsi à des succès de recrutement différents entre espèces pondant à différentes périodes de l'année (STRANGE et al., 1992). En revanche, le succès de recrutement de certaines années permet l'obtention de larges cohortes (CARREL et RIVIER, 1996 ; TAYLOR et al., 1996). A titre d'exemple, en 1992, les densités de truite fario de la station 1410 ont été multipliées par 5 par rapport à l'année précédente, avec $90 \%$ de l'effectif total constitué de juvéniles (0+). GROSSMAN et al. (1990) suggèrent que les YOY (Young Of the Year) présentent une structure stochastique liée à la forte variabilité des conditions environnementales, tandis que dans le cas des adultes les facteurs biotiques sont prépondérants et les peuplements sont alors plus stables. Nous n'avons pas pu tester cette hypothèse, car nous ne disposons pas de données sur les différentes classes d'âge des espèces. Quoi qu'il en soit, les crues et les étiages ont également une incidence sur les adultes en raison notamment des modifications physiques de l'habitat et des abris disponibles pour les poissons (GORMAN, 1986 ; FREEMAN et al., 1988 ; PUSEY et al., 1993 ; TAYLOR et al., 1996). Malgré le fait que certaines espèces se remettent facilement de ce type de perturbations externes, leur taux de récupération (" recovery rate ") dépend de facteurs tels que les conditions hydrologiques (ROSS et al., 1985), la durée et l'intensité des perturbations (ROSS et al., 1985), la capacité des espèces à surmonter ces perturbations et la capacité des survivants à repeupler le milieu (ROSS et al., 1985 ; MEFFE et BERRA, 1988), la présence de zones refuges (ROSS et al., 1985 ; STRANGE et al., 1992 ; PUSEY et al., 1993), la tolérance physiologique des espèces (ROSS et al., 1985 ; TAYLOR et al., 1996).

Dans le cas des peuplements de poissons des cours d'eau bas-normands, il n'y a pas de relation apparente entre la persistance des espèces et la variabilité temporelle du peuplement (Figure 3). L'hypothèse de HORWITZ (1978), stipulant que les fortes variabilités qui touchent le peuplement induisent un turnover important des espèces qui le constituent, n'est donc pas vérifiée. HORWITZ (1978) ajoute que les peuplements situés à l'amont d'un cours d'eau sont moins stables que ceux situés en aval, car sujets aux importantes variations des conditions environnementales caractéristiques de ces régions, et sont donc plus voués à l'extinction. Or dans notre cas, nous obtenons une relation inverse : la variabilité temporelle des peuplements est positivement corrélée à celle des débits (Figure 2), qui est elle-même plus importante dans les zones d'aval (Figure 4). Une partie des cours d'eau considérés naît de résurgences des nappes phréatiques régulant ainsi, en permanence, les débits à l'amont. Cette caractéristique pourrait expliquer les différences de variabilité des débits entre l'amont et l'aval.

Quel que soit l'indice de variabilité temporelle utilisé $(C V, W)$, la stabilité des peuplements considérés diminue lorsque l'on augmente le nombre d'années prises en compte (Tableau II). Ces résultats corroborent ceux obtenus par HUGUENY et al. (1995) et rejoignent ceux de PIMM et REDFEARN (1988) et de BENGTSSON et al. (1997) sur des populations d'oiseaux et d'insectes.

BENGTSSON et al. (1997) proposent 4 explications possibles à l'augmentation de la variabilité temporelle des peuplements :

1. autocorrelations entre les densités des populations pour les années adjacentes. En augmentant la durée du suivi, on couvre plus d'un turnover des espèces et la variabilité environnementale augmente la variabilité temporelle. Cette caractéristique ne s'applique pas à notre étude puisqu'elle ne couvre en moyenne qu'un changement de génération ; 
2. sachant que la variabilité environnementale augmente au cours du temps, si les populations locales répondent aux fluctuations locales en augmentant leur variabilité au cours du temps et si les espèces répondent individuellement à la variabilité environnementale, on observera de la sorte des différences d'abondances relatives d'autant plus importantes que la durée de suivi des peuplements est importante ;

3. à l'échelle régionale, les tendances à long terme qui touchent l'abondance des différentes espèces, en réponse aux changements environnementaux, se répercutent sur les peuplements à l'échelle locale ;

4. l'échantillonnage aléatoire (l'échelle spatiale utilisée), bien que censé être le plus représentatif des peuplements, tient une place non négligeable dans la variabilité observée.

Les hypothèses 2 et 3 sont compatibles avec nos résultats. En effet, chacune des espèces qui compose les peuplements étudiés réagit de façon différentielle aux variations environnementales (différence dans : les périodes de ponte et de recrutement, la distribution sur le gradient vertical, les habitats, la taille des individus et leurs vagilités...) et contribue de la sorte à faire augmenter la variabilité du peuplement à mesure que l'on augmente la durée du suivi. Par ailleurs, à l'échelle locale, les peuplements de poissons des cours d'eau semblent dépendre, en partie, de processus qui s'opèrent à l'échelle régionale (HUGUENY et PAUGY, 1995 ; BELKESSAM et al., 1997 ; OBERDORFF et al., 1998).

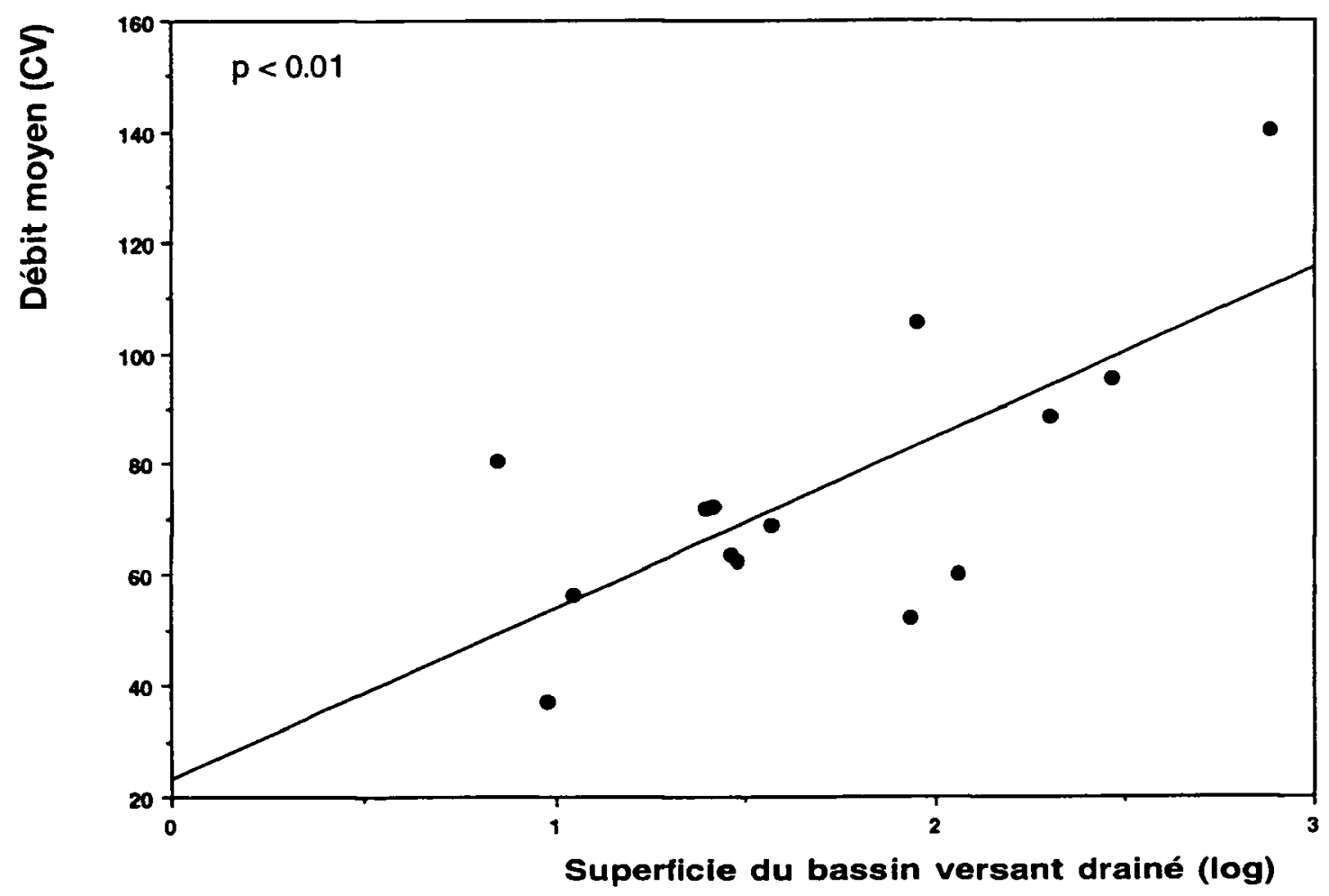

Figure 4

Relation entre les $\mathrm{CV}$ des débits moyens et la superficie du bassin versant drainé.

Figure 4

Relationship between mean flows $\mathrm{CV}$ and the surface area of the drainage basin. 


\section{CONCLUSION ET PERSPECTIVES}

Les peuplements de poissons des cours d'eau bas-normands sont persistants et ont une structure relativement stable $(W)$. Ils présentent néanmoins une importante variabilité temporelle au niveau des densités des espèces (CVDM) déterminée, en partie, par les variations des facteurs hydrologiques (débits). On peut penser que, face à ces variations des facteurs hydrologiques, les espèces adoptent des stratégies variables (FREEMAN et al., 1988 ; TAYLOR et al., 1996). II serait donc maintenant intéressant d'analyser pour chaque espèce les relations entre leur variabilité temporelle et celle de chacun des facteurs hydroclimatiques : “ des événements environnementaux imprévisibles peuvent conduire à des variations prévisibles de la structure des peuplements pour peu que l'on sache comment les différentes espèces réagissent aux variations des conditions environnementales » (YANT et al., 1984).

\section{REMERCIEMENTS}

Nous tenons à remercier le Conseil Supérieur de la Pêche et la DIREN Basse-Normandie pour avoir mis à notre disposition les diverses données. Nos remerciements vont également à $\mathrm{B}$. Hugueny et à deux lecteurs anonymes pour leurs conseils.

\section{BIBLIOGRAPHIE}

BELKESSAM D., OBERDORFF T., HUGUENY B., 1997. Unsaturated fish assemblages in rivers of the North-Western France : potential consequences for species introductions. Bull. Fr. Pêche Piscic., 344/345, 193-204.

BENGTSSON J., 1994. Temporal predictability in forest soil communities. Journal of Animal Ecology, 63, 653-665.

BENGTSSON J., BAILLIE S.R., LAWTON J., 1997. Community variability increases with time. Oikos, 78, 249-256.

CARLE F.L., STRUB M.R., 1978. A new method for estimating population size from removal data. Biometrics, 34, 621-630.

CARREL G., RIVIER B., 1996. Distribution of three euryoecious cyprinids in the main channel of the Lower River Rhône. Arch. Hydrobiol., 113, 363-374.

DIAMOND J.M., MAY R.M., 1977. Species turnover rates on islands : dependence on census interval. Science, 197, 266-270.

FREEMAN M.C., CRAWFORD M.K., BARRETT J.C., FACEY D.E., FLOOD M.G., HILL J., STOUDER D.J., GROSSMAN G.D., 1988. Fish assemblage stability in a southern Appalachian stream. Canadian Journal of Fisheries and Aquatic Sciences, 45, 1949-1958.

GATZ A.J., 1979. Community organization in fishes as indicated by morphological features. Ecology, 60, 711-718.

GORMAN O.T., KARR J.R., 1978. Habitat structure and stream fish communities. Ecology, 59, 507-515.

GORMAN O.T., 1986. Assemblage organization of stream fishes : the effect of rivers on adventitious streams. Am. Nat., 128, 611-616.

GROSSMAN G.D., MOYLE P.B., WITHAKER J.O., 1982. Stochasticity in structural and functional characteristics of an Indiana stream fish assemblage : a test for community theory. Am. Nat., 120, 423-454. 
GROSSMAN G.D., FREEMAN M.C., MOYLE P.B., WITHAKER J.O., 1985. Stochasticity and assemblage organization in an Indiana stream fish assemblage. Am. Nat., 126, 275-285.

GROSSMAN G.D., DOWD J.F., CRAWFORD M., 1990. Assemblage stability in streams : a review. Environmental Management, 14, 661-671.

HERBOLD B., 1984. Structure of an Indiana stream fish association : choosing an appropriate model. Am. Nat., 124, 561-572.

HORWITZ R.J., 1978. Temporal variability patterns and the distributional patterns of stream fishes. Ecol. Monogr., 48, 307-321.

HUGUENY B., 1992. Onchocerciasis control programm in West Africa. Fish monitoring data analysis. OCP/VCU/HYBIO., 92.4, 2 p.

HUGUENY B., PAUGY D., 1995. Unsaturated fish communities in African rivers. Am. Nat, 146, 162-169.

HUGUENY B., PERSAT H., BAGLINIĖRE J.L., BOËT P., CARREL G., OLIVIER J.M., PAUGY D., PONT D., TRAORÉ K., 1995. Variabilité à long terme des peuplements de poissons : résultats provenant de rivières françaises et ouest-africaines. Bull. Fr. Pêche Piscic., 337/338/339, 93-99.

KENNEDY G.J.A., PITCHER T.J., 1975. Experiments on homing in shoals of the European minnows, Phoxinus phoxinus (L.). Transactions of the American Fisheries Society, 104, 454-457.

MEFFE G.K., BERRA T.M., 1988. Temporal characteristics of fish assemblage structure in an Ohio stream. Copeia, 3, 684-690.

MINNS C.K., 1995. Allometry of home range size in lake and river fishes. Canadian Journal of Fisheries and Aquatic Sciences, 52, 1499-1508.

MOYLE P.B., VONDRACEK B., 1985. Persistence and structure of the fish assemblage in a small Californian stream. Ecology, 66, 1-13.

NICOLAS Y., PONT D., LAMBRECHTS A., 1994. Using $\gamma$-emitting artificial radionuclides, released by nuclear plants, as markers of restricted movements by chub, Leuciscus cephalus, in a large river, the lower Rhône. Environmental Biology of Fishes, 39, 399-409.

OBERDORFF T., HUGUENY B., COMPIN A., BELKESSAM D., 1998. Non-interactive fish communities in the coastal streams of North-Western France. Journal of Animal Ecology, 67, 472-484.

PIMM S., REDFEARN A., 1988. The variability of population densities. Nature, 334, 613614.

PIMM S., 1991. The balance of nature ? University of Chicago Press, Chicago, USA.

PUSEY B.J., ARTHINGTON A.H., READ M.G., 1993. Spatial and temporal variation in fish assemblage structure in the Mary river, south-eastern Queensland : the influence of habitat structure. Environmental Biology of Fishes, 37, 355-380.

RAHEL F.J., LYONS J.D., COCHRAN P.A., 1984. Stochastic or deterministic regulation of assemblage structure? It may depend on how the assemblage is defined. Am. Nat., 124, 583-589.

ROSS S.T., MATTHEWS W.J., ECHELLE A.A., 1985. Persistence of stream fish assemblages : effects of environmental changes. Am. Nat., 126, 24-40.

SCHLOSSER I.J., 1982. Fish community structure and function along two habitat gradients in a headwater stream. Ecol. Monogr., 52, 395-414.

SCHLOSSER I.J., 1987. A conceptual framework for fish communities in small warmwater streams. In MATTHEWS W.J., D.C. HEINS (eds), Community and evolutionary ecology of North American stream fishes. University of Oklahoma Press, 17-24. 
SCHLOSSER I.J., 1990. Environmental variation, life history attributes, and community structure in stream fishes : implications for environmental management and assessment. Environ. Manage., 14, 621-628.

SIEGEL S., 1956. Nonparametric statistics for the behavioral sciences. McGraw-Hill Kogakusha Ltd, New York.

STRANGE E.M., MOYLE P.B., FOIN T.C., 1992. Interactions between stochastic and deterministic processes in stream fish community assembly. Environmental Biology of Fishes, 36, 1-15.

TAYLOR C.M., WINSTON M.R., MATTTHEWS W.J., 1996. Temporal variation in tributary and main stream fish assemblages in Great Plains stream system. Copeia, 2, 280289.

VERNEAUX J., 1977. Biotypologie de l'écosystème « eau courante ». Détermination approchée de l'appartenance typologique d'un peuplement ichtyologique. C. $R$. Acad. Sc. (Paris), 284, 675-678.

VERNEAUX J., 1981. Les poissons et la qualité des cours d'eau. Annales Scientifiques de l'Université de Franche-Comté, 2, 33-41.

YANT P.R., KARR J.R., ANGERMEIER P.L., 1984. Stochasticity in stream fish communities : an alternative interpretation. Am. Nat., 124, 573-582. 